\title{
Kernos
}

Revue internationale et pluridisciplinaire de religion grecque antique

$29 \mid 2016$

Varia

\section{Hosios. A Semantic Study of Greek Piety}

\section{Philippe Borgeaud}

\section{CpenEdition \\ Journals}

\section{Édition électronique}

URL : http://journals.openedition.org/kernos/2419

DOI : 10.4000/kernos.2419

ISSN : 2034-7871

\section{Éditeur}

Centre international d'étude de la religion grecque antique

\section{Édition imprimée}

Date de publication : 1 octobre 2016

Pagination : 424-428

ISSN : 0776-3824

Référence électronique

Philippe Borgeaud, "Hosios. A Semantic Study of Greek Piety », Kernos [En ligne], 29 | 2016, mis en ligne le 01 octobre 2016, consulté le 17 novembre 2020. URL : http://journals.openedition.org/kernos/ 2419 ; DOI : https://doi.org/10.4000/kernos.2419

Ce document a été généré automatiquement le 17 novembre 2020.

Kernos 


\title{
Hosios. A Semantic Study of Greek Piety
}

\author{
Philippe Borgeaud
}

\section{RÉFÉRENCE}

Saskia PEELS, Hosios. A Semantic Study of Greek Piety, Leiden/Boston, Brill, 2016. 1 vol. 1 vol. 16,5 × 24,5 cm, 295 p. (Mnemosyne supplements. Monographs on Greek and Roman Language and Literature, 387). ISBN : 978-90-04-29463-9.

1 Dans le Cyclope d'Euripide, Ulysse se plaint de la mauvaise rencontre: "Hélas! J'ai surmonté les épreuves de Troie et de la mer, et voici que je tombe sur un homme anhósios, au cœur sauvage et inabordable !» Qu'est-ce qui fait, précisément, dans cette scène, de Polyphème un anhósios (qualificatif généralement traduit par « impie ») ? Estce son mépris affiché pour les dieux, et même pour son père Poséidon, ou simplement le traitement cannibale qu'il réserve à ses hôtes ou suppliants? La réponse (que je ne trahirai pas ici) sera donnée au terme d'une lumineuse et rigoureuse analyse, menée par Saskia Peels (S.P.) dans un ouvrage qui devrait renouveler, voire relancer les études sur le vocabulaire grec du "sacré " ${ }^{1}$, même si «sacré » est un terme que l'auteure, prudemment, évite le plus possible ou met entre guillemets. Comme le titre de son livre l'indique, elle préfère parler de " piété » (en pensant à eusébeia).

2 Anhósios, c'est le contraire de hósios. Hósios et les lexèmes qui lui sont apparentés (227 occurrences littéraires et 11 épigraphiques, entre Homère et le début du $\mathrm{IV}^{\mathrm{e}} \mathrm{s}$. av. n.è.) renvoient à la notion de " piété ». Mais sous quel angle exactement ? L'étymologie ne donne aucune réponse fiable. Mieux vaut donc s'adresser aux champs sémantiques. Et, de ce point de vue, ces lexèmes apparaissent pour le moins fuyants (« elusive »). Le même mot renvoie parfois à des qualités très proches de celles que désignent aussi hagnós, díkaios ou encore eusebés : pureté, justice, piété semblent se recouper. Mais sontelles à envisager dans les rapports entre humains et dieux, ou simplement entre humains ? Là aussi, les champs se recoupent. 
3 La première difficulté abordée par S.P. sera par conséquent celle de déterminer si hósíos et les termes apparentés désignent plutôt une appréciation morale (un point de vue purement humain, voire politique), ou un jugement « religieux » supposé exprimer le point de vue des dieux. Cette question a fait l'objet d'un ancien débat, qu'elle résume, entre Van der Valk et Bolkestein avant d'être reposée par Benveniste, Rudhardt et Maffi (cf. note supra). Pour Robert Parker², hósios désigne un espace neutre, de «normalité », à l'abri des dangers du sacré et de la pollution. Or, cet espace abrite aussi bien des personnes que des comportements ou des objets. Dans ce dernier cas (celui des objets), on traduit souvent hósios par « profane».

4 Partant de ces questions préliminaires, il s'agira aussi de comprendre ce que signifie la popularité croissante de hósios au $\mathrm{v}^{\mathrm{e}} \mathrm{s}$. av. n.è. Ce changement de régime correspond-il à un changement de "mentalité " ? Saskia Peels est ainsi entraînée à poser la question du rapport entre religion politique et individuelle, en examinant (entre autres) les textes où la naturalisation d'un étranger est définie comme l'octroi des hierà et hósia de la cité. Mais l'essentiel de son enquête (les chapitres 1 à 4, p. 1 à 148) porte sur des questions concernant la polysémie apparente du lexème hósios et son rapport sémantique à des plus ou moins synonymes (eusebés, et díkaios).

Pour aborder ces questions de sémantique, l'auteure a recours aux instruments de la linguistique cognitive, qu'elle expose de manière limpide (en citant ses sources). Elle postule tout d'abord que le choix d'un lexème pour exprimer un message est une affaire de compétition entre une série de lexèmes plus ou moins proches les uns des autres. Un choix s'opère donc au sein d'un lexique mental correspondant à un inventaire de situations possibles. Deux lexèmes ne peuvent pas s'équivaloir, mais leurs champs sémantiques peuvent partiellement se recouper. Dans la théorie linguistique classique remontant à Aristote, la signification d'un mot renvoie à une catégorie clairement définie par des traits nécessaires et suffisants; cette catégorie est donc strictement délimitée, distincte de ses voisines. Les expériences réalisées par la psychologie ont cependant montré qu'il s'agit là d'un idéal théorique. Ce n'est pas comme cela que les humains, dans la réalité de leur vécu, pensent les catégories. Un objet, dans la perception que l'on s'en fait, peut appartenir «plus ou moins" à telle ou telle catégorie. Il y a, dans l'expérience vécue et son rendu verbal, une flexibilité sémantique dont doit tenir compte toute analyse lexicale. Un même usage peut être " prototypique » pour un lexème, et « marginal » pour un autre. Il y a aussi des usages, c'est-à-dire des choix, plus ou moins fréquents ou préférentiels, selon les contextes ou les structures d'expérience (les cadres, «frames»). Pour identifier l'usage «prototypique » d'un lexème, il ne faut pas se fier à la fréquence des usages, mais (quand la documentation le permet) observer quel est le mot qui semble venir spontanément à la bouche d'un locuteur, avant tout raisonnement, toute théorisation.

6 Dans la grande majorité de leurs occurrences hósios et anhósios sont utilisés pour évaluer, exprimer un jugement positif ( $36 \%)$ ou négatif $(60 \%)$ sur un comportement humain, généralement le comportement d'un autre. Ce jugement porte sur le rapport aux dieux ( $24 \%$ ), à la maisonnée ( $23 \%$ ), aux morts (13\%), aux hôtes ou aux suppliants (14\%), au respect d'un serment (13\%). S'agit-il ici d'éthique ou de «religion»? La question, ainsi posée, n'est pas la bonne. Il s'agit en effet de l'une et de l'autre à la fois (p. 45), comme l'auteure le montre à partir des usages de ces termes depuis Homère, jusqu'à l'Euthyphron de Platon. A moins, me permettrai-je de suggérer, qu'il ne s'agisse 
encore d'autre chose comme le montre implicitement l'ensemble de ce livre, où l'opposition du « religieux » à ce qui ne le serait pas s'estompe de page en page.

Il n'en reste pas moins que le substantif tò hósion en vient, au ve s., à signifier ce que les humains doivent faire pour plaire aux dieux et leur donner la timè qu'ils méritent. Il ne faudrait pas conclure trop vite, de cette évolution lexicale, à un changement de «mentalité »: ce que hósios et les lexèmes apparentés désignent à l'époque classique était en effet déjà signifié, à l'époque archaïque, par thémis et díke (p. 67).

8 Il faut donc chercher une autre raison qu'un tournant mental, pour expliquer l'évolution lexicale de hósios et apparentés au $\mathrm{v}^{\mathrm{e}} \mathrm{s}$. La réponse, selon l'auteure, passe par l'examen rigoureux des rapports explicitement exprimés dans les textes, entre hósios et eusebés (p. 68-106)3 , et entre hósios et díkaios (p. 107-148). On a parfois suggéré que l' eusébeia serait une question de croyance relative à l'existence et à la nature des dieux, tandis que l'hosiótes (la qualité d'hósios) concernerait la pratique conventionnelle. La première serait alors une disposition innée, la seconde consisterait finalement à soutenir, en le respectant par des actions adressées aux dieux, un ordre social. Les choses ne sont malheureusement pas si simples : les emplois des deux termes renvoient quasiment aux mêmes contenus. Mais anhósios est employé beaucoup plus souvent qu' asebès avec une coloration violemment négative, pour disqualifier l'attitude d'un impie. Le contraste établi par les Grecs entre les deux groupes de termes porte donc moins sur les représentations (les concepts qu'ils désignent), que sur leur charge affective (p. 85); un maintien calme et rationnel, convenant à la famille d'eusebès, s'oppose à l'agitation émotionnelle évoquée par la famille d'hósios (p.99). S.P. démontre cela de manière convaincante, en analysant des passages emblématiques de l'Héraclès d'Euripide, de l' Edipe-roi de Sophocle, des Suppliantes et des Sept contre Thèbes d'Eschyle, dont elle donne chaque fois des traductions très précises.

9 Avec dikaios, le contraste est différemment orienté. Dans l'Odyssée et parfois jusqu'à Pindare, dike commente ce qui obéit à la coutume; mais plus tard dike en vient à signifier ce qui est juste sous l'angle des procédures juridiques. La comparaison entre hósios et díkaios conduit S.P. à examiner de près le contexte des dialogues tragiques impliquant des suppliants. Le choix d'hosíos et des lexèmes apparentés, plutôt que de dike et apparentés, apparaît dans ce cadre relever d'une rhétorique de stratégie argumentative. La multiplication des occurrences d'hósios au $\mathrm{V}^{\mathrm{e}} \mathrm{s}$. ainsi que l'introduction, à la même époque, d'eusebès et des lexèmes apparentés sont probablement liées, selon l'auteure, à ce changement sémantique de dike, qui nous oriente vers les procédures d'attaque et de défense, au tribunal. C'est ainsi par exemple que dans l'ÆEdipe à Colonne de Sophocle les emplois d'hósios et de dikaios dans les discours concernant la supplication d'Ædipe semblent renvoyer les premiers aux aspects plus "religieux» de l'asile accordé aux suppliants, les seconds à ce qui est permis aux humains par la loi (extrader le suppliant, ou le considérer comme appartenant à la cité). La dikè, il faut le souligner, est ambigüe; elle se présente différemment en fonction de qui s'arroge le droit de juger et de rendre une sentence : ceux qui viennent réclamer le suppliant en ont une autre conception que ceux qui veulent l'accueillir. Cela est montré de manière particulièrement claire dans la lecture que S.P. fait des Suppliantes d'Eschyle. Et surtout, elle est définie tantôt comme qualifiant un comportement que la partie adverse, à l'instar des dieux, devrait percevoir comme juste, tantôt, simplement, comme un comportement rigoureusement adéquat à la règle juridique. Une telle multiplicité de signification peut entraîner des malentendus. Il se 
peut donc que la popularité croissante d'hósios et anhósios, corrélative à l'introduction d'eusebès et de sa famille, ait pour cause le besoin de disposer d'un plus riche vocabulaire.

10 Dans la dernière partie de son livre (les chapitres 5 à 7), l'auteure aborde quelques problèmes plus spécifiques, et tout d'abord les usages d'hósios ou des lexèmes apparentés pour qualifier des comportements divins. L' «impie» Apollon de l'Oreste d'Euripide est certes surprenant, comme l'est aussi le «pieux» Apollon de son Alceste, l'Apollon amoureux qui s'interroge sur la légitimité (hosía) de sa passion pour la nymphe Cyrène, ou enfin Zeus, dont la même Alcmène (dans l'Héraclès) se demande s'il s'est bien comporté avec elle. Pour commenter les occurrences d'hósios et anhósios qualifiant des divinités, S.P. fait appel à la théorie linguistique des marques. Tandis que Rudhardt expliquait ces occurrences en faisant l'hypothèse que les dieux sont obligés, comme les humains, de se conformer à un ordre supérieur à eux, elle les explique comme des exceptions paradoxales, visant parfois un effet comique, en déplaçant le dieu sur un plan humain. C'est ainsi qu'elle comprend, aussi, les occurrences d'hosíe dans l'Hymne homérique à Hermès.

11 Le chapitre 6 aborde les occurrences des termes de la famille d'hósios (notamment ouk hósion, marquant un interdit particulièrement redouté) dans le dossier épigraphique et aussi littéraire des règlements rituels. L'inscription d'Ialysos (Rhodes) (LSCG 136) fait l'objet d'une lecture poussée, à propos des prescriptions concernant des animaux dans le contexte d'une refondation du culte. Le règlement s'adresse d'abord à des étrangers, qui pourraient transgresser par ignorance des règles particulièrement importantes pour la communauté locale. La comparaison s'imposait avec un règlement de Cos (LSCG 150 a), concernant l'interdiction de couper du bois dans l'enceinte sacrée. L'auteure aborde enfin un groupe d'inscriptions proférant des interdit (oukh hósion ou ou thémis) contre les femmes, pour des raisons « religieuses ». Elle constate finalement que l'usage des termes asébeia et oukh hósion / hosía dans les textes rituels normatifs est fort rare ; il est motivé par la grande importance, pour la cohésion de telle ou telle communauté, d'une règle particulière qu'un étranger pourrait négliger.

Pour son dernier chapitre (p. 207-251), S.P. choisit d'affronter l'épineux (" highly perplexing») problème de la supposée possible traduction (que proposent les principaux dictionnaires) d'hósios tantôt en sacré, tantôt en profane. Les monnaies " sacrées » (hierá chrémata) qui appartiennent aux dieux sont en effet parfois dites hósia. Il en va de même des humains. Van der Valk expliquait cela par deux étapes, sur une ligne temporelle : quand un humain accomplit ses devoirs envers les dieux, il est hósios dans le sens de sacré et pieux ; quand il retourne à ses activités normales, après avoir été libéré de ses obligations religieuses, il est hósios dans le sens de profane. Il en irait de même des monnaies appartenant aux dieux, quand elles sont rendues à l'usage profane. Henri Jeanmaire, prolongeant cet argument, proposait que hosíe, dans l'Hymne homérique à Hermès, aurait signifié une part sacrificielle désacralisée ${ }^{4}$. Pour des raisons linguistiques (l'extrême rareté des "auto-antonymes » ou "contranymes »), et aussi encouragée par des remarques de Rudhardt, S.P. préfère, a priori, renoncer à cette explication (souvent partagée). Elle relève aussi, à l'instar encore de Rudhardt, que l'opposition sacré/profane, introduite par Durkheim (et non par Robertson Smith : pace Bremmer, cité note 23, p. 2115) ne convient pas au monde grec. Elle se réfère à Maffi et Connor (cf. note supra), qui ont insisté sur le caractère homogène de ce qu'on pourrait désormais (en référence aux travaux des ethnologues ${ }^{6}$ ) appeler ici encore une 
«ontologie » grecque, pour laquelle le monde est infusé de divin. En choisissant de qualifier d'hósia telles ou telles monnaies, la cité signale que l'usage de cet argent à des fins spécifiques plaît aux dieux.

S.P., selon une méthode où elle excelle, propose des analyses de textes pour approcher de la solution (la peste d'Athènes vue par Thucydide, le rituel des chevaux d'Onchestos dans l'Hymne homérique à Apollon, un passage de la Lysistrata d'Aristophane, quelques vers de l'Iphigénie en Tauride et des Bacchantes d'Euripide). Elle montre qu'on peut chaque fois renoncer à l'idée de "profane " pour expliquer ces textes. Elle peut alors s'attaquer aux occurrences d'hosíe dans l'Hymne homérique à Hermès. Après avoir discuté les interprétations proposées du fameux passage où Hermès offre aux dieux une hosie de viande rôtie, en étant tenté d'en manger lui-même, elle remarque que l'auteur de l' Hymne n'avait pas l'intention de décrire un rituel cohérent. Il s'agit, selon elle, d'un jeu poétique construit en référence à l'épisode prométhéen de la Théogonie hésiodique. Hermès prépare un repas pour les dieux, comme si son action était située dans le temps d'avant la séparation entre hommes et dieux. Avec la référence à hosíe et le refus d'Hermès de manger, la scène se trouve soudain située après la dispute et la séparation. L'épisode décrit dans l'Hymne à Hermès est donc une mise en scène répétant, de manière plaisante, le moment de la séparation entre dieux et humains à Mékonè ; dans ce jeu la perspective des uns comme des autres est incarnée par Hermès (ce garnement qui est un immortel, mais qui fait " comme » s'il était un mortel).

En conclusion, retenons qu'on ne rencontre que de très rares coquilles («Hermion » pour « Hermione » p. 137, «Godulier » pour «Godelier» p. 265), et signalons l'usage parfois peu judicieux des termes «religieux » (opposé à ce qui ne le serait pas?), et " mentalité » (dans le sens de représentation mentale). Je relèverai que la plus grande qualité de ce livre, à part les solutions toujours intéressantes qu'il propose (et que l'on pourra bien sûr discuter), est sa clarté. Je souligne enfin que la méthode suivie mériterait d'inspirer de nouvelles visites de dossiers non moins épineux, comme, pour n'en citer qu'un seul, celui des usages (qui se recoupent parfois) de religio et superstitio en latin.

\section{NOTES}

1. .Parmi d'autres: J.C. BOLKESTEIN, Hósios en Eusebés: Bijdrage tot de Godsdienstige en Zedelijke Terminologie van de Grieken, Amsterdam, 1936 ; M.H.A.L.H. Van der Valk, «Zum Worte HOSIOS », Mnemosyne 10 (1943), p.113-140; E. BENVENISTE, Le vocabulaire des institutions indo-européennes, vol. 2, Paris, 1969, p. 192-207 ; A. MAFFI, « Ta hierà kaì tà hósia. Contributo al hosíos lo studio della terminologia giuridico-sacrale greca », in J. MODRZEJEWSKI, D. LIEBS (éd.), Vorträge zur griechischen und hellenistischen Rechtsgeschichte. Symposion Chantilly 1977, Cologne, 1982, p.\$-\$; A. MOTTE, "L'expression du sacré dans la religion grecque», in J. RIES (éd.), L'expression du sacré dans les grandes religions III, Louvain-la-Neuve, 1986, p. 109-256 ; W.R. CONNOR, « Sacred and Secular. Hierà kaì hósia and the Classical Athenian Concept of the State ", Ancient Society 19 (1988), p. 161-188 ; J. RUDHARDT, Notions fondamentales de la pensée religieuse et actes constitutifs du culte dans la Grèce 
classique, Paris, Picard, $1992^{2}$ (avec une importante seconde préface); L. BRUIT ZAIDMAN, Le Commerce des dieux. Eusebeia. Essai sur la piété en Grèce ancienne, Paris, 2001 ; G. JAY-ROBERT, Le Sacré et la loi. Essai sur la notion d'hosion d'Homère à Aristote, Paris, 2009 ; J.H. BLOK, « Hosiê and Athenian law from Solon to Lykourgos ", in V. AZOULAY, P. ISMARD (éd.), Clisthène et Lycurgue d'Athènes. Autour du politique dans la cité classique, Paris, 2011, p. 233-254.

2. .R. PARKER, Miasma: Pollution and Purification in Early Greek Religion, Oxford, $1996^{2}$, p. 12, 330, 338.

3. Pour eusebès et eusébeia, et surtout pour sébas, l'A. se réfère entre autres à la thèse de Rudhardt sur la piété athénienne (cf. note supra), ainsi qu’à ses Opera inedita posthumes (Kernos, suppl. 19, Liège, 2008) ; elle aurait aussi pu tirer profit d'un article paru, il est vrai, dans un volume relativement obscur : "Considérations sur la notion de sebas ", in Homère chez Calvin. Mélanges Olivier Reverdin, Genève, 2000, p. 421-434.

4. .H. JEANMAIRE, «Le substantif hosia et sa signification comme terme technique dans le vocabulaire religieux », REG 58 (1945), p. 66-89.

5. .Cf. Ph. BORGEAUD, «Le couple sacré/profane. Genèse et fortune d'un concept "opératoire" en histoire des religions », RHR 211 (1994), p. 387-418.

6. .Cf. A.I. HALlOWELL, « Ojibwa Ontology, Behavior, and World View, in R.D. FOGELSON et al. (éd.), Contributions to Anthropology. Selected Papers of A. Irving Hallowell, Chicago, 1976, p. 357-390; Ph. DESCOLA, Par-delà nature et culture, Paris, Gallimard, 2005. Cf. M. SAHLins, The Western Illusion of Human Nature, Chicago, 2008.

\section{AUTEURS}

\section{PHILIPPE BORGEAUD}

Université de Genève 